\title{
Lipid-poor renal angiomyolipoma: Differentiation from clear cell renal cell carcinoma using wash-in and washout characteristics on contrast-enhanced computed tomography
}

\author{
PINGKUN XIE ${ }^{1 *}$, ZHIHUI YANG ${ }^{2 *}$ and ZHENG YUAN ${ }^{3}$ \\ ${ }^{1}$ Department of Radiology, Ningbo Beilun People's Hospital, Ningbo, Zhejiang 315800; \\ ${ }^{2}$ Department of Pathology, 81 st Hospital of the People's Liberation Army (PLA), Nanjing, Jiangsu 210000; \\ ${ }^{3}$ Department of Radiology, Shanghai PLA No. 85 Hospital, Shanghai 200052, P.R. China
}

Received January 11, 2015; Accepted January 26, 2016

DOI: $10.3892 / 01.2016 .4214$

\begin{abstract}
In the present study, a total of 82 patients (42 men and 40 women; age range, 24-84 years), including 34 patients with lipid-poor renal angiomyolipoma (AML) and 49 with clear cell renal cell carcinoma (RCC), who had undergone multiphase contrast-enhanced computed tomography (CT) (i.e., CT with unenhanced, corticomedullary, nephrographic and 5-min delay phase scanning) were evaluated. The peak enhancement attenuation value, net enhancement attenuation value, enhancement ratio, washout value and washout ratio were calculated to compare the enhancement characteristics between the two diseases. The results revealed that the lipid-poor AMLs had a significantly higher mean attenuation value compared with that of CCRCCs on unenhanced CT scans (37.8 vs. $30.9 \mathrm{HU}$; Mann-Whitney U test, $\mathrm{P}=0.003$ ). In addition, significant differences were found between lipid-poor AMLs and CCRCCs with regard to wash-in (Mann-Whitney U test, $\mathrm{P}=0.001$ ) and enhancement ratios (Mann-Whitney $\mathrm{U}$ test, $\mathrm{P}=0.010$ ) on contrast-enhanced CT scans. A receiver operating characteristic (ROC) analysis revealed an area under the curve (AUC) of 0.722 using wash-in for differentiation between CCRCCs and lipid-poor AMLs. Lipid-poor AMLs exhibited a reduced washout of contrast enhancement $(35.8 \pm 32.7 \mathrm{HU}$ washout value; $29.4 \pm 0.187 \%$ washout ratio) compared with that of CCRCCs $(48.3 \pm 28.4 \mathrm{HU}$ washout value; $35.7 \pm 0.148 \%$ washout ratio; Mann-Whitney $\mathrm{U}$ test, $\mathrm{P}=0.037$ and $\mathrm{P}=0.204$, respectively). The ROC analysis result yielded an AUC of 0.639 for the use of washout to differentiate CCRCCs from
\end{abstract}

Correspondence to: Dr Zheng Yuan, Department of Radiology, Shanghai PLA No. 85 Hospital, 1328 Huashan Road, Shanghai 200052, P.R. China

E-mail: yuanzheng0404@163.com

*Contributed equally

Key words: renal angiomyolipoma, clear cell renal cell carcinoma, lipid-poor, washout, computed tomography, X-ray lipid-poor AMLs. In summary, a larger wash-in and washout of contrast enhancement is a predictor that a lesion is CCRCC.

\section{Introduction}

Renal angiomyolipoma (AML) is the most common type of benign solid renal tumor and may be diagnosed by computed tomography (CT) imaging, relying on the detection of macroscopic fat with negative attenuation measurements (1). However, CT examination is unable to detect intratumoral fat content in $\sim 4.5 \%$ of AMLs, including minimal fat and lipid-poor AMLs (1). On unenhanced CT images, the appearance of lipid-poor AML has been described as homogeneous and hyperattenuating compared with its surrounding renal parenchyma (1). However, such hyperattenuation is nonspecific: Other renal masses, such as metastases, venous infarctions, leiomyomas and $22 \%$ of clear cell renal cell carcinomas (CCRCCs) also exhibit this feature on CT images $(2,3)$. Patients with lipid-poor AML typically receive unnecessary surgery for suspected renal cell carcinoma (RCC) when a diagnosis is not specifically established prospectively. Various strategies have been proposed to differentiate lipid-poor AML from RCC on the basis of imaging characteristics. These approaches have included attenuation measurement histogram analysis (4-7), analysis of contrast enhancement patterns (3), and chemical-shift magnetic resonance imaging (8).

A previous study reported that biphasic helical CT imaging may be useful in differentiating lipid-poor AML from RCC, with homogeneous tumor enhancement and prolonged enhancement patterns being the most valuable CT findings (3). Several studies have attempted to assess the washout characteristics of adrenal and pulmonary lesions on contrast-enhanced CT, and this is suggested to be helpful in differentiating malignant from benign tumors (9-13). Washout refers to the reduction of the attenuation values of lesions on CT during a variable period subsequent to the intravenous injection of a bolus of contrast material (12). To the best of our knowledge, no evaluation of the washout characteristics of lipid-poor AML and CCRCC on dynamic contrast-enhanced CT has been reported. The purpose of the present study was to compare the wash-in and washout characteristics of lipid-poor 
AML and CCRCC, and to assess the potential clinical value of wash-in and washout values on dynamic CT scanning for the differentiation of lipid-poor AML from CCRCC.

\section{Materials and methods}

Patients. Between September 2009 and September 2011, a total of 96 patients with renal masses underwent dynamic enhanced CT examination at Shanghai PLA No. 85 Hospital (Shanghai, China) and Ningbo Beilun People's Hospital (Ningbo, China). From this, 82 patients (42 men and 40 women; age range, 24-84 years; mean age, 53.0 \pm 13.6 years; median age, 55 years) with a total of 83 lesions had been pathologically confirmed to have either lipid-poor AML (34 AMLs in 33 patients, with no identifiable macroscopic fat on CT images) or CCRCC (49 tumors in 49 patients). Patients were excluded according to the following criteria: (i) Absence of surgical pathological results; (ii) presence of identifiable macroscopic fat on CT images.

CT examinations. Four-phase CT examinations were performed on a 64-slice multidetector CT scanner (LightSpeed VCT; GE Medical Systems, Milwaukee, WI, USA) or a dual-source CT scanner (Somatom Definition; Siemens AG, Medical Solutions, Forchheim, Germany). A 90-ml volume of nonionic contrast agent [iopamidol (Iopamiro ${ }^{\circledR} 370$ ); Bracco Imaging S.p.A., Milan, Italy] was administered by a power injector at a rate of $3 \mathrm{ml} / \mathrm{sec}$. The scanning protocol included data acquisition in four phases: The unenhanced phase, the corticomedullary phase (30-sec delay following contrast injection), the nephrographic phase (90-sec delay following contrast injection) and the delay phase (5-min delay following contrast injection. The scanning parameters were as follows: Pitch, 0.625; X-ray tube voltage, $120 \mathrm{kV}$; and tube current, 200-400 mA. The slice thickness of axial and coronal images was $5 \mathrm{~mm}$. Coronal multiplanar reconstruction imaging was routinely performed for the corticomedullary and nephrographic phases.

Image analysis. Renal mass enhancement on dynamic CT images was measured on circular, operator-defined regions of interest (ROIs) on the selected image for each cluster at each time point (i.e., from the unenhanced image to the image acquired at 5-min). Each ROI, covering 50-80\% of the tumor surface area, was examined. Large ROIs were selected in order to incorporate solid-appearing parts of a tumor, and to exclude obvious cystic or necrotic areas.

From the mean Hounsfield unit (HU) value in each ROI of the renal mass on the dynamic and delayed CT scans, the peak enhancement attenuation value, net enhancement attenuation value (wash-in), enhancement ratio, and absolute and relative loss of enhancement (washout value and washout ratio) were calculated. The wash-in value was calculated by subtracting the pre-enhancement attenuation value from peak enhancement attenuation value. Washout value was calculated by subtracting the attenuation value at 5-min from peak enhancement attenuation value. Washout ratio was calculated as follows: 1 - (attenuation value at 5-min / peak enhancement attenuation value) $\mathrm{x} 100 \%$.

Threshold values were retrospectively used to define different types of time-attenuation curves: Type A with wash-in
$<70 \mathrm{HU}$ and any washout; type B with persistent enhancement (no washout); type $\mathrm{C}$ with wash-in $\geq 70 \mathrm{HU}$ and washout $>50 \mathrm{HU}$; and type D with wash-in $\geq 70 \mathrm{HU}$ and washout $\leq 50 \mathrm{HU}$.

Statistical analysis. Statistical analysis was performed using SPSS software (version 10.0; SPSS, Inc., Chicago, IL, USA). Statistical analyses of the differences between each enhancement characteristic in lipid-poor AMLs and CCRCCs were performed with the Mann-Whitney U test. Receiver operating characteristic (ROC) analysis was performed to evaluate the usefulness of the unenhanced CT attenuation, net enhancement and washout values as markers for differentiating CCRCCs from AMLs. The area under the ROC curve (AUC) was calculated, and ranged from 0.5 to 1.0 , increasing when diagnostic performance approached that of the reference standard (in this study, determination of CCRCCs). The $\chi^{2}$ test or Fisher's exact test were used to analyze statistically significant time-attenuation curve differences between lipid-poor AML and CCRCC. Two-sided tests were used and $\mathrm{P}<0.05$ was considered to indicate a statistically significant difference.

\section{Results}

Of the 83 renal masses, $49(59.0 \%)$ proved to be CCRCCs and $34(41.0 \%)$ proved to be lipid-poor AMLs. The mean diameter of the renal masses was $4.6 \pm 2.2 \mathrm{~cm}$ (range, $1.5-10.0 \mathrm{~cm}$; median, $4.5 \mathrm{~cm}$ ). The mean attenuation value of lipid-poor AMLs (mean $37.8 \pm 14.8 \mathrm{HU}$; range 6.7-58.0 HU) on unenhanced CT scans was significantly higher than that of CCRCCs (mean 30.9 7.4; range 15.8-48.9 HU) (Mann-Whitney U test, $\mathrm{P}=0.003$ ). The ROC analysis result revealed an AUC of 0.674 using the attenuation value on unenhanced CT scans for differentiating CCRCCs from lipid-poor AMLs. The threshold of $\leq 37 \mathrm{HU}$ determined for the attenuation value on unenhanced $\mathrm{CT}$ scans was associated with a sensitivity of $78.7 \%$ and specificity of $62.5 \%$.

Early enhancement CT and wash-in of contrast material. The characteristics of the enhancement dynamics of renal masses on early phase contrast-enhanced CT are summarized in Table I. The net enhancement attenuation (wash-in) of the 34 lipid-poor ALMs on early contrast-enhanced CT scans was $70.9 \pm 43.4 \mathrm{HU}$, and enhancement ratio was $62.8 \pm 19.2 \%$ (Fig. 1). The net enhancement attenuation (wash-in) of the 49 CCRCCs on enhanced CT scans was $96.2 \pm 35.6 \mathrm{HU}$, and enhancement ratio was $73.7 \pm 9.3 \%$ (Fig. 2). Significant differences were observed between lipid-poor AMLs and CCRCCs with regard to the net enhancement attenuation (wash-in) (Mann-Whitney $\mathrm{U}$ test, $\mathrm{P}=0.001$ ) and enhancement ratio (Mann-Whitney $\mathrm{U}$ test, $\mathrm{P}=0.010)$ on contrast-enhanced $\mathrm{CT}$ scans. The ROC analysis yielded an AUC of 0.722 for the use of wash-in for differentiating CCRCCs from lipid-poor AMLs. The threshold value of $\geq 84 \mathrm{HU}$ determined for wash-in was associated with sensitivity of $61.7 \%$ and specificity of $81.2 \%$.

Delayed contrast-enhanced $C T$ and relative percentage washout of contrast material. Lipid-poor AMLs exhibited a reduced washout of contrast enhancement (washout value, $35.8 \pm 32.7 \mathrm{HU}$; washout ratio, $29.4 \pm 0.187 \%$ ) compared with that of CCRCCs (washout value, $48.3 \pm 28.4 \mathrm{HU}$; washout ratio, 35.7 $\pm 0.148 \%$ ) (Mann-Whitney $\mathrm{U}$ test, $\mathrm{P}=0.037$ and 
Table I. Lesion characteristics on early enhancement computed tomography.

\begin{tabular}{|c|c|c|c|}
\hline Characteristic & Clear cell renal cell carcinoma $(n=49)$ & Renal angiomyolipoma ( $\mathrm{n}=34)$ & P-value ${ }^{a}$ \\
\hline Pre-enhancement value (HU) & & & 0.003 \\
\hline Mean \pm SD & $30.9 \pm 7.4$ & $37.8 \pm 14.8$ & \\
\hline Median & 30.1 & 40.5 & \\
\hline Range & $15.8-48.9$ & $6.7-58.0$ & \\
\hline Net enhancement value (HU) & & & 0.001 \\
\hline Mean \pm SD & $96.2 \pm 35.6$ & $70.9 \pm 42.7$ & \\
\hline Median & 94.5 & 60.7 & \\
\hline Range & $18.5-177.3$ & $15.8-195.1$ & \\
\hline Enhancement ratio (\%) & & & 0.010 \\
\hline Mean \pm SD & $73.7 \pm 9.3$ & $62.8 \pm 18.9$ & \\
\hline Median & 75.1 & 63.7 & \\
\hline Range & $52.7-89.2$ & 21.4-89.8 & \\
\hline
\end{tabular}

HU, Hounsfield units; SD, standard deviation. ${ }^{\mathrm{a} M a n n-W h i t n e y ~ U ~ t e s t . ~}$

Table II. Patterns of nodule enhancement at early and delayed enhancement computed tomography.

\begin{tabular}{lcrr}
\hline & \multicolumn{2}{c}{ Number of lesions } \\
\cline { 2 - 4 } Pattern of enhancement & $\begin{array}{c}\text { Clear cell renal } \\
\text { cell carcinoma (n=49) }\end{array}$ & $\begin{array}{c}\text { Renal } \\
\text { angiomyolipoma (n=34) }\end{array}$ \\
\hline Type A: wash-in $<70$ HU and any washout & 9 & 14 & 0.022 \\
Type B: persistent enhancement (no washout) & 1 & 2 & $<0.001$ \\
Type C: wash-in $\geq 70$ HU and washout $>50$ HU & 19 & 6 & 0.001 \\
Type D: wash-in $\geq 70$ HU and washout $\leq 50$ HU & 19 & 039 \\
\hline
\end{tabular}

HU, Hounsfield units. ${ }^{\mathrm{a}} \chi^{2}$ test.

$\mathrm{P}=0.204$, respectively) (Figs. 1 and 2). The ROC analysis result revealed an AUC of 0.639 for the use of washout to differentiate CCRCCs from lipid-poor AMLs. The threshold value of $\geq 40 \mathrm{HU}$ determined for washout was associated with a sensitivity of $61.7 \%$ and specificity of $62.5 \%$.

Types of time-attenuation curves. The most common type of time-attenuation curve for CCRCCs was type C ( $\geq 70 \mathrm{HU}$ wash-in and $>50$ HU washout) (20/49 lesions; 40.8\%), whilst for lipid-poor ALMs, type A (<70 HU wash-in and any washout) was the most common (14/34 lesions; $41.2 \%)$. Type B (no washout) occurred in $2.0 \%$ of CCRCCs (1/49 lesions) and $35.3 \%$ of lipid-poor ALMs (12/34 lesions) $\left(\chi^{2}\right.$ test, $\left.\mathrm{P}<0.001\right)$ Type D ( $\geq 70 \mathrm{HU}$ wash-in and $\leq 50 \mathrm{HU}$ washout) occurred in $38.8 \%$ of CCRCCs (19/49 lesions) and $8.8 \%$ of lipid-poor ALMs (3/34 lesions) ( $\chi^{2}$ test, $\left.\mathrm{P}=0.039\right)$ (Table II).

\section{Discussion}

Solid renal masses lacking macroscopic fat remain a diagnostic dilemma. Various strategies have been proposed to differentiate lipid-poor AML from RCC on the basis of imaging characteristics (4-8). In the current study, the results indicated that the combination of wash-in and washout characteristics on contrast-enhanced multi-detector row CT may be useful for the differentiation of CCRCC from lipid-poor AML.

The definition of lipid-poor AML is generally established on the basis of findings on unenhanced CT scans; however, such tumors are actually observed to have intratumoral fat upon microscopic examination (4). Previous studies reported that high tumor attenuation in the unenhanced phase may be a finding specific to lipid-poor AML $(4,14,15)$, whereas CCRCC has a lower unenhanced phase attenuation (16). In the present study, the mean attenuation value of AMLs $(37.8 \pm 14.8 \mathrm{HU})$ on unenhanced CT scans was significantly higher than that of CCRCCs (mean, 30.9 7.4 HU) (Mann-Whitney U test, $\mathrm{P}=0.003$ ). The ROC analysis showed that the AUC is 0.674 using the attenuation value on unenhanced CT scans for differentiating CCRCCs from AMLs.

Multiphasic contrast enhancement is another method that researchers have proposed for the differentiation of lipid-poor AML from RCC. However, previous studies have produced variable results $(3,16)$, thus limiting the utility of this approach. These conflicting results may predominantly stem from the different subtypes of RCC, which exhibit varying enhancement patterns. A previous study has also demonstrated that 

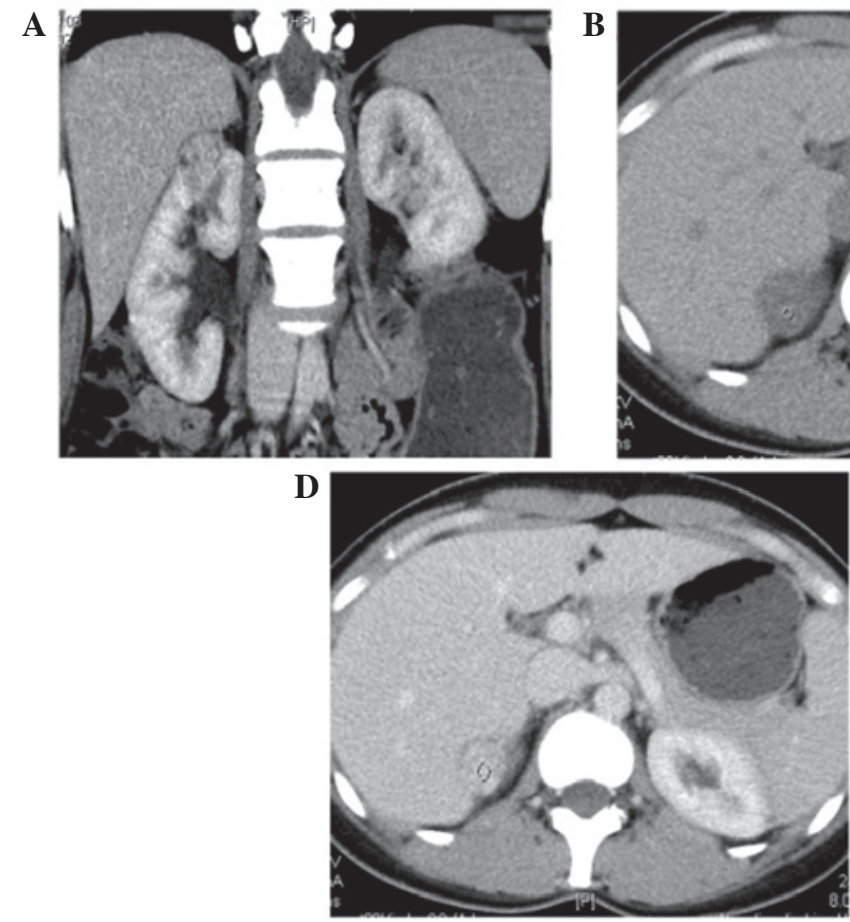
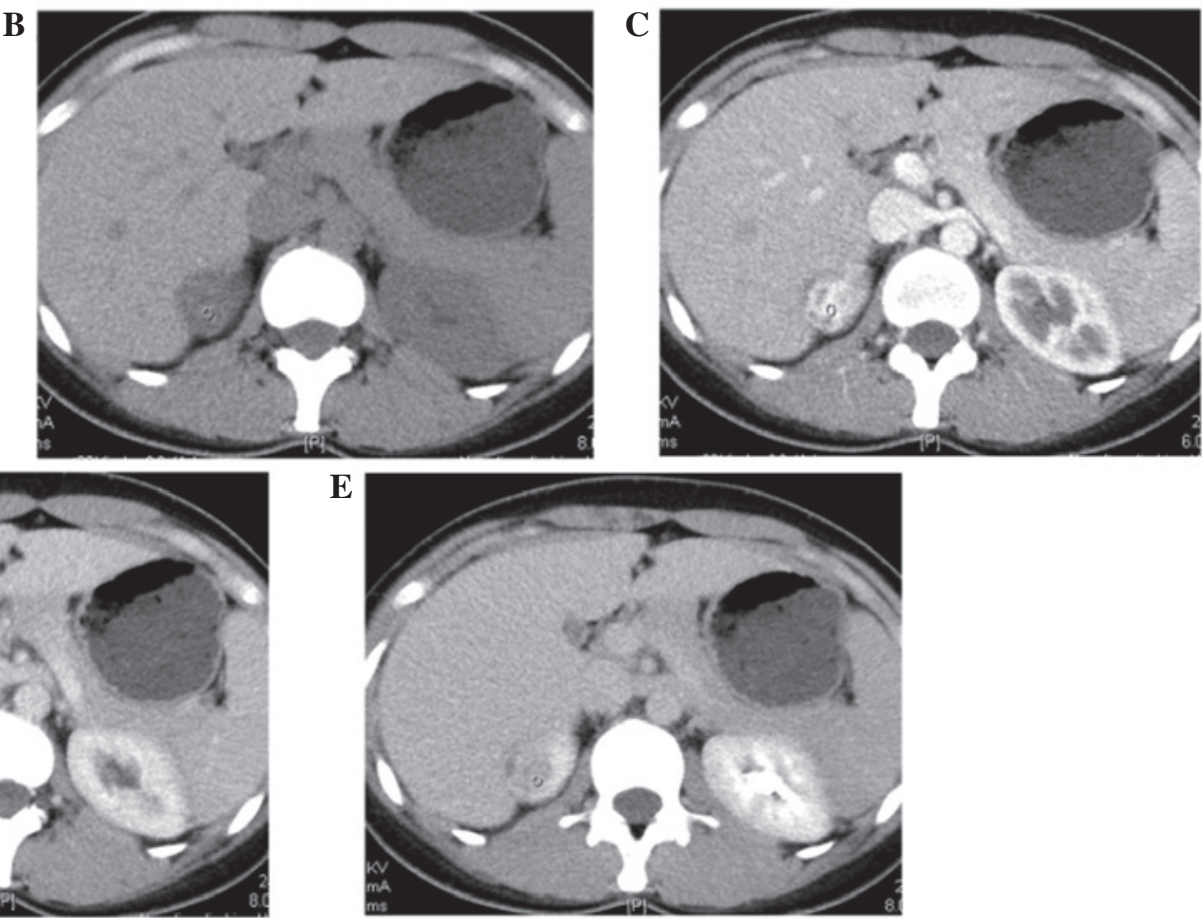

Figure 1. CT scans of (A) lipid-poor angiomyolipoma in a 29-year-old man in the right upper pole of kidney. (B) The CT attenuation value on the non-enhanced scan was $42 \mathrm{HU}$. (C) The enhancement value was $103 \mathrm{HU}$ at $30 \mathrm{sec}$ on enhancement CT scan, and (D) $99 \mathrm{HU}$ at $90 \mathrm{sec}$. (E) At 5 min on the enhancement CT scan, the CT attenuation value was $66 \mathrm{HU}$. The wash-in and washout values are $61 \mathrm{HU}$ and $37 \mathrm{HU}$ respectively. CT, computed tomography; HU, Hounsfield units.

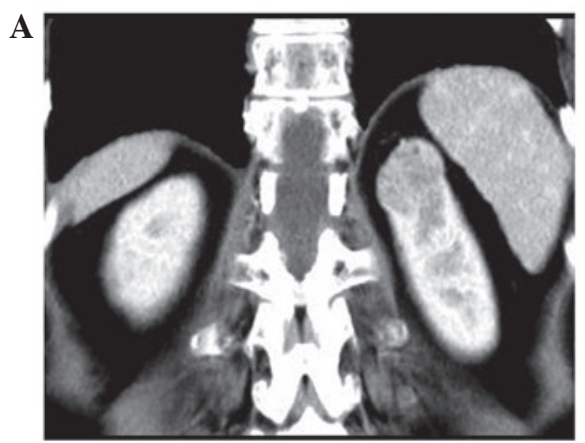

D

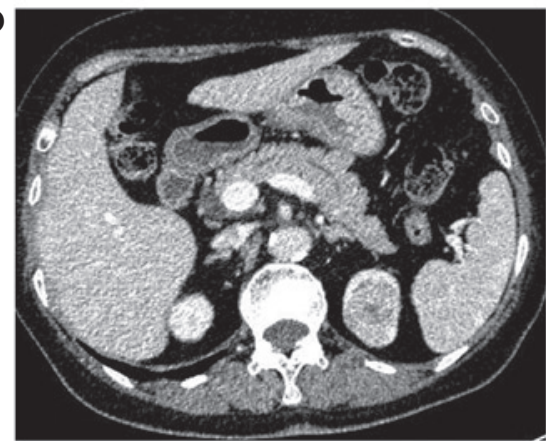

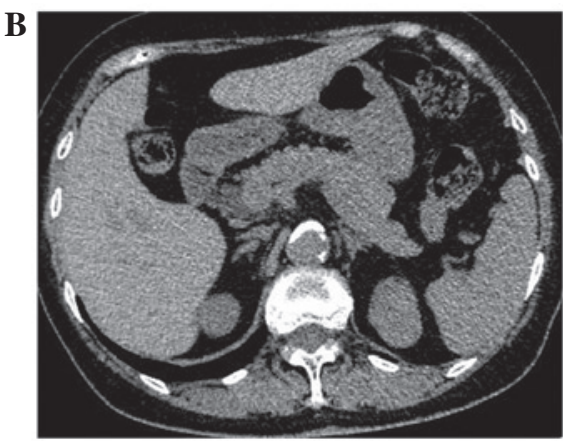
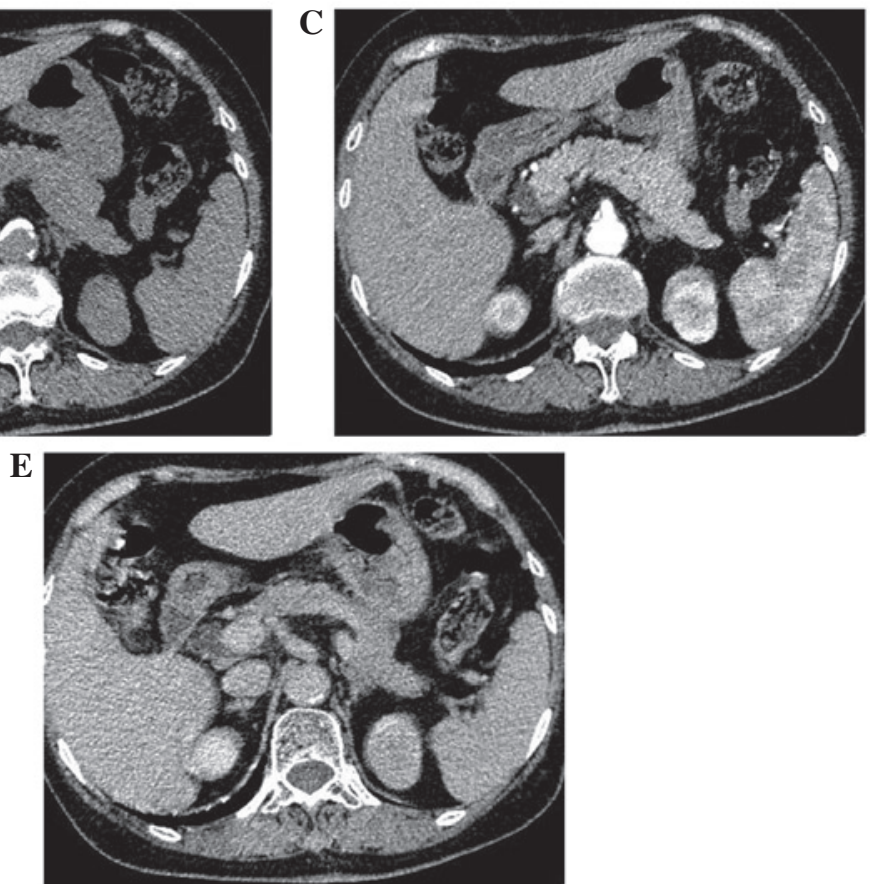

Figure 2. CT scans of (A) clear cell renal cell carcinoma in the left upper pole of the kidney of a 78-year-old woman. (B) The CT attenuation value on the non-enhanced scan was $39 \mathrm{HU}$. (C) The enhancement value was $173 \mathrm{HU}$ at $30 \mathrm{sec}$ on the enhancement CT scan and (D) $96 \mathrm{HU}$ at $90 \mathrm{sec}$. (E) At $5 \mathrm{~min}$ on the enhancement CT scan, the CT attenuation value was $72 \mathrm{HU}$. The wash-in and washout were $134 \mathrm{HU}$ and $101 \mathrm{HU}$ respectively. CT, computed tomography; HU, Hounsfield units.

homogeneous enhancement and a prolonged enhancement pattern were the most valuable CT findings for differentiating lipid-poor AML from RCC (3). In the current study, in general, CCRCCs tended to enhance substantially more than lipid-poor AMLs. Net enhancement attenuation (wash-in) of CCRCCs on early contrast-enhanced $\mathrm{CT}$ scans was higher than that of AMLs (96.2 vs. $70.9 \mathrm{HU}, \mathrm{P}=0.001)$. The ROC analysis revealed an AUC of 0.722 using net enhancement attenuation (wash-in) for differentiating CCRCCs from lipid-poor AMLs. Using a cut-off value of $84 \mathrm{HU}$ net enhancement, the 
diagnostic sensitivity for CCRCCs is $61.7 \%$, and specificity is $81.2 \%$. The washout of contrast enhancement on 5 -min contrast-enhanced CT scans was also higher in CCRCCs than in lipid-poor AMLs (48.3 vs. $35.8 \mathrm{HU}, \mathrm{P}=0.037$ ). The ROC analysis result showed AUC is 0.639 using washout of contrast enhancement on 5-min contrast-enhanced CT scans for differentiating CCRCCs from lipid-poor AMLs. Using a cut-off value of $40 \mathrm{HU}$ net enhancement, the diagnostic sensitivity for CCRCCs is $61.7 \%$ and specificity is $62.5 \%$.

The biological basis for the observed differences in wash-in and washout characteristics between CCRCCs and lipid-poor AMLs may be postulated as follows. The bio-distribution of nonspecific contrast medium is determined by the relative level of vascular perfusion of different tissues and their capillary permeability (12). The majority of malignant tumors have a high level of vascular perfusion and a large extracellular space, which would contribute to intense enhancement in early enhanced CT scans (12). As for the majority of AMLs, distorted blood vessel and blood sinusoids would contribute to the retention of contrast medium in delay enhanced CT scans (15). This explanation may apply to CCRCCs with intense enhancement and larger washout than lipid-poor AMLs on multiphasic contrast-enhanced CT scans.

In conclusion, the differential diagnosis of CCRCCs and lipid-poor AMLs may be achieved using wash-in and washout characteristics on contrast-enhanced CT. Larger wash-in and washout values of contrast enhancement are predictors that a lesion is CCRCC. However, the sensitivity and specificity of the dynamic CT in the current study were not high enough; thus, further studies, particularly involving multivariate analysis, are required.

\section{Acknowledgements}

This study was partly supported by the Science Technology Commission of Shanghai Municipality (grant no. 124119a0100) and the National Natural Science Foundation of China (grant nos. 81301218 and 81301262).

\section{References}

1. Choyke PL, Glenn GM, Walther MM, Zbar B and Linehan WM: Hereditary renal cancers. Radiology 226: 33-46, 2003.
2. Hafron J, Fogarty JD, Hoenig DM, Li M, Berkenblit R and Ghavamian R: Imaging characteristics of minimal fat renal angiomyolipoma with histologic correlations. Urology 66: $1155-1159,2005$

3. Kim JK, Park SY, Shon JH and Cho KS: Angiomyolipoma with minimal fat: Differentiation from renal cell carcinoma at biphasic helical CT. Radiology 230: 677-684, 2004.

4. Simpfendorfer C, Herts BR, Motta-Ramirez GA, Lockwood DS, Zhou M, Leiber M and Remer EM: Angiomyolipoma with minimal fat on MDCT: Can counts of negative-attenuation pixels aid diagnosis? AJR Am J Roentgenol 192: 438-443, 2009.

5. Simpson E and Patel U: Diagnosis of angiomyolipoma using computed tomography-region of interest $<$ or $=-10 \mathrm{HU}$ or 4 adjacent pixels $<$ or $=-10 \mathrm{HU}$ are recommended as the diagnostic thresholds. Clin Radiol 61: 410-416, 2006.

6. Kim JY, Kim JK, Kim N and Cho KS: CT histogram analysis: Differentiation of angiomyolipoma without visible fat from renal cell carcinoma at CT imaging. Radiology 246: 472-479, 2008

7. Catalano OA, Samir AE, Sahani DV and Hahn PF: Pixel distribution analysis: Can it be used to distinguish clear cell carcinomas from angiomyolipomas with minimal fat? Radiology 247: 738-746, 2008.

8. Kim JK, Kim SH, Jang YJ, Ahn H, Kim CS, Park H, Lee JW, Kim S and Cho KS: Renal angiomyolipoma with minimal fat: Differentiation from other neoplasms at double-echo chemical shift FLASH MR imaging. Radiology 239: 174-180, 2006.

9. Jeong YJ, Lee KS, Jeong SY, Chung MJ, Shim SS, Kim H, Kwon OJ and Kim S: Solitary pulmonary nodule: Characterization with combined wash-in and washout features at dynamic multidetector row CT. Radiology 237: 675-683, 2005.

10. Pena CS, Boland GW, Hahn PF, Lee MJ and Mueller PR: Characterization of indeterminate (lipid-poor) adrenal masses: Use of washout characteristics at contrast-enhanced CT. Radiology 217: 798-802, 2000.

11. Caoili EM, Korobkin M, Francis IR, Cohan RH, Platt JF, Dunnick NR and Raghupathi KI: Adrenal masses: Characterization with combined unenhanced and delayed enhanced CT. Radiology 222: 629-633, 2002.

12. Ye XD, Ye JD, Yuan Z, Dong S and Xiao XS: Characterization of solitary pulmonary nodules: Use of washout characteristics at contrast-enhanced computed tomography. Oncol Lett 3: 672-676, 2012

13. Ye XD, Ye JD, Yuan Z, Li WT and Xiao XS: Dynamic CT of solitary pulmonary nodules: Comparison of contrast medium distribution characteristic of malignant and benign lesions. Clin Transl Oncol 16: 49-56, 2014.

14. Jinzaki M, Tanimoto A, Narimatsu Y, Ohkuma K, Kurata T, Shinmoto H, Hiramatsu K, Mukai $M$ and Murai M: Angiomyolipoma: Imaging findings in lesions with minimal fat. Radiology 205: 497-502, 1997.

15. Silverman SG, Mortele KJ, Tuncali K, Jinzaki M and Cibas ES: Hyperattenuating renal masses: Etiologies, pathogenesis and imaging evaluation. Radiographics 27: 1131-1143, 2007.

16. Zhang J, Lefkowitz RA, Ishill NM, Wang L, Moskowitz CS, Russo P, Eisenberg H and Hricak H: Solid renal cortical tumors: Differentiation with CT. Radiology 244: 494-504, 2007. 